\title{
Session 6
}

\section{[ICMID18-26AE] Potential Use of Pomalaa Nickel Slag as A Substitute for Sand in Brick Making}

\author{
E Ngii, B Mursidi and Y Y Umar \\ Department of Civil Engineering, Faculty of \\ Engineering, Universitas Halu Oleo \\ E-mail: edward.ngii@uho.ac.id
}

\begin{abstract}
The latest variance of nickel slag from PT.Antam Pomalaa has material gradation size equivalent to sand has, so it has potentially as a sand substitute material. The study aims to determine the use of fine nickel slag as a substitute for sand in brick making. Nickel slag used is variation of $0 \%$, $25 \%, 50 \%, 75 \%$ and $100 \%$ against of total weight of sand. The brick is designed with a cement - sand ratio of $1: 7$. The exprimental is a hollow brick with dimension of $40 \mathrm{~cm} \times 18 \mathrm{~cm} \times 10 \mathrm{~cm}$. Compressive test is done after brick curing along 28 days. The maximum result of compressive test is obtained in the use of $50 \%$ nickel slag of $19.58 \mathrm{~kg} / \mathrm{cm}^{2}$ compared to the brick without nickel slag of $12.92 \mathrm{~kg} / \mathrm{cm}^{2}$. The conclusion that by using a fine nickel slag type of fenil iv up to $50 \%$ on production, it can reduce sand use and improve the strength up to $51 \%$.
\end{abstract}




\section{CERTIFICATE}

Faculty of Engineering, Universitas Halu Oleo certifies the participation of

\section{Edward Ngii}

as

Presenter

at the 1st International Conference on

Maritime Infrastructure and Development (ICMID)

held in Kendari, South East Sulawesi, Indonesia

on 19-21 November 2018

Dean,

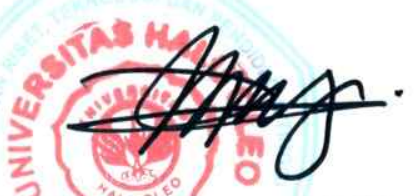

Dr. Edward Ngii

Chairperson of The Organizing Committee,

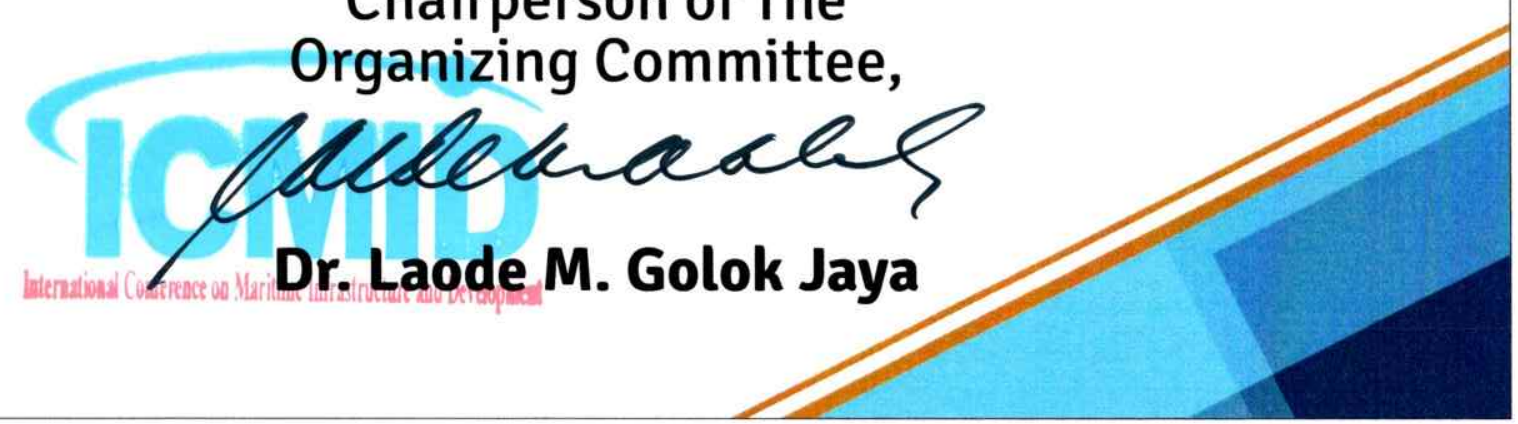


THE IST INTERNATIONAL CDNFERENCE QN MARITIME INFRASTRUCTURE AND DEVELIPMENT (ILMID) ZQI8
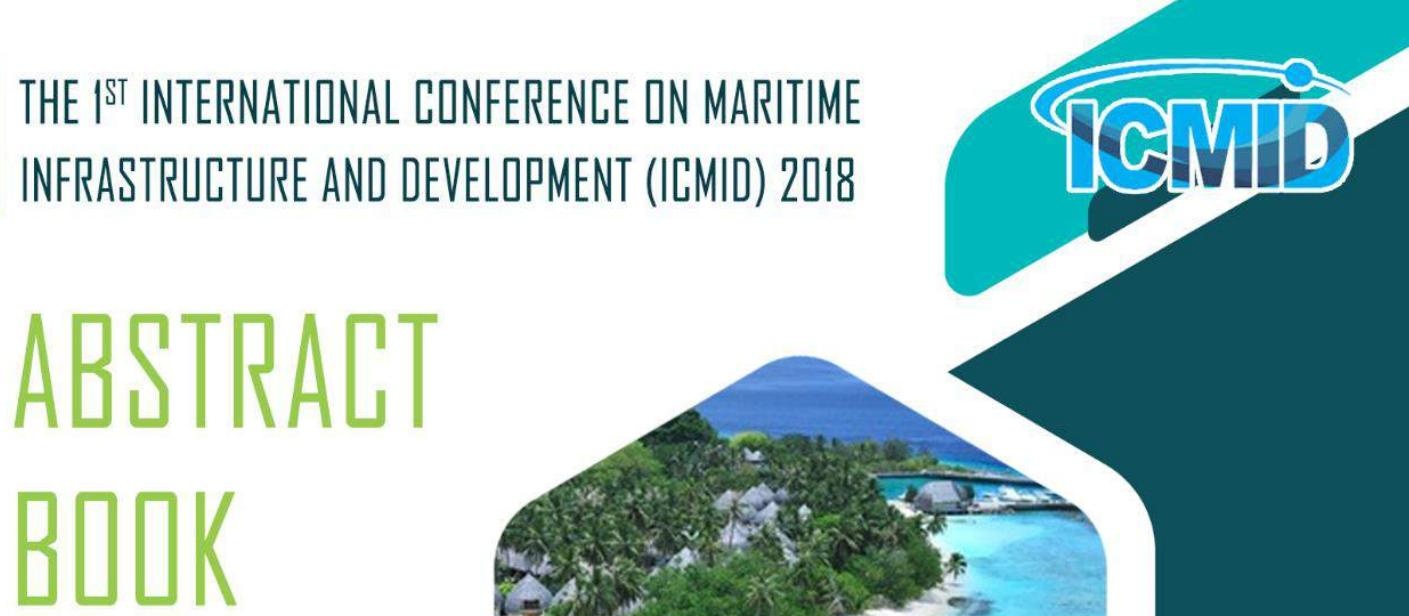


\section{Foreword from ICMID Chairperson}

First of all I would like to welcome all of our distinguish guest, speakers, authors and participants who make contribution in the ICMID international seminar at the Faculty of Engineering, University of Halu Oleo (UHO). Welcome to Kendari, the capital of Southeast Sulawesi Province. I hope you will enjoy stay in Kendari during this seminar.

As the largest archipelagic country in the world, Indonesia has great potential to become the world's maritime axis. The maritime axis is a strategic idea that is realized to ensure inter-island connectivity, the development of the shipping and fisheries industry, the improvement of sea transportation and a focus on maritime security. Indonesia has a fundamental interest in mastering and utilizing the sea because Indonesia has a strong maritime identity and culture in utilizing its strategic position to create security in the region in accordance with the mandate of the constitution.

To realize this, the synergy between academics and researchers, the government and the business world is needed to make a major contribution to the development of programs for the development of Indonesia's maritime infrastructure. This ICMID international seminar seeks to make a positive contribution to achieving this goal.

We have invited researchers and practitioners to contribute ideas in scientific papers that will be presented in two days. There are 57 papers which are collaborations of authors from various countries and universities. 57 of these papers varied from aspects of science, applied engineering and policy science. We sincerely hope that the results of the research outlined in the paper presented at this seminar will benefit the Indonesian and world maritime development. 
ICMID 2018

Kendari, Indonesia 19-20 November 2018

I would like to express thank to Rector of Universitas Halu Oleo Professor Muhammad Zamrun and Dean of Faculty of Engineering Dr. Edward Ngii who support this conference. I specially thank to all of organizing committee who have make great contribution and support to manage this seminar.

Thank you and congratulations on conducting the seminar.

Kendari, 19 November 2018

Chairperson of the ICMID,

Dr. Laode M. Golok Jaya 


\section{Foreword from Dean of Faculty of Engineering UHO}

I welcome the implementation of this ICMID International Seminar. I also conveyed welcome to all delegations from various countries and universities. I hope you all enjoy the natural beauty of Kendari as part of the Indonesian archipelago, beautiful and charming maritime continent.

Halu Oleo University (UHO) Kendari as part of the nation's community has a strong vision of maritime development. This is reflected in UHO's vision, namely in 2045 to become the 250 leading universities in the world in science and technology for the continent of maritime, harmonization and sustainable prosperity.

To realize the vision of UHO 2045 specifically related to maritime affairs, UHO needs to develop maritime research in the form of basic research, applied research and development. UHO researchers need to collaborate with and collaborate with other researchers from both domestic institutions and universities.

The UHO Faculty of Engineering as the core of applied research and development in UHO in the field of technology needs to work together with various parties, especially with the government, business world and society in supporting the acceleration of maritime infrastructure development. The UHO Faculty of Engineering has a great responsibility to integrate and collect various expertise resources that exist both internally and externally to jointly develop maritime research. The acceleration of collaborative research between researchers and the government as policy makers and the business world and the community as users of research results is needed to support Indonesia's maritime vision. Therefore, I am very happy that the Faculty of Engineering UHO can hold an international seminar to accelerate the collaboration of 
ICMID 2018

Kendari, Indonesia 19-20 November 2018

researchers from home and abroad in all fields related to maritime affairs.

I hope this international seminar can continue in the future to build a positive contribution to the development of the Indonesian maritime continent. Enjoy the seminar.

Kendari, 19 November 2018

Dean,

Dr. Edward Ngii 


\title{
Technical Program
}

\author{
Program at-a-Glance
}

\section{November 19, 2018 | Monday}

Grand Clarion Hotel \& Convention Kendari, Southeast Sulawesi, Indonesia 07:30-08:30 Registration Participants Registration \& Conference Kits

Collection

\begin{tabular}{|c|c|c|c|}
\hline & \multicolumn{2}{|c|}{$\begin{array}{c}\text { November 19, } 2018 \text { | Monday } \\
\text { Grand Clarion Hotel \& Convention Kendari }\end{array}$} & Room \\
\hline 08:30-08:40 & \multicolumn{2}{|c|}{$\begin{array}{l}\text { Welcome performance by Mondotambe Traditional } \\
\text { Dancing }\end{array}$} & Ball Room \\
\hline $08: 40-08: 50$ & \multicolumn{2}{|c|}{ Indonesia Raya National Anthem } & Ball Room \\
\hline 08:50-09:00 & $\begin{array}{l}\text { ICMID } \\
\text { Report }\end{array}$ & $\begin{array}{l}\text { Dr. L.M. Golok Jaya } \\
\text { (Chairperson of The Organizing } \\
\text { Committee ICMID 2018) }\end{array}$ & Ball Room \\
\hline 09:00-09:10 & $\begin{array}{l}\text { Welcome } \\
\text { Speech }\end{array}$ & $\begin{array}{l}\text { Dr. Edward Ngii } \\
\text { (Dean of Faculty of Engineering } \\
\text { UHO) }\end{array}$ & Ball Room \\
\hline 09:10-09:30 & $\begin{array}{l}\text { Opening } \\
\text { Speech }\end{array}$ & $\begin{array}{l}\text { Prof. Muh. Zamrun } \\
\text { (Rector of Universitas Halu Oleo) }\end{array}$ & Ball Room \\
\hline 09:30-09:45 & $\begin{array}{l}\text { Honorary } \\
\text { Speech }\end{array}$ & $\begin{array}{l}\text { Mr. H. Abu Hasan, M.Pd. (Regent } \\
\text { of North Buton) }\end{array}$ & Ball Room \\
\hline $09: 45-10.00$ & \multicolumn{3}{|c|}{ Group Photo \& Coffee Break } \\
\hline $10: 00-10: 45$ & $\begin{array}{l}\text { Keynote } \\
\text { Speech I }\end{array}$ & $\begin{array}{l}\text { Luhut Binsar Pandjaitan, MPA } \\
\text { (Minister of maritime affairs } \\
\text { coordinator, Republic of } \\
\text { Indonesia) }\end{array}$ & Ball Room \\
\hline $10: 45-11: 30$ & $\begin{array}{l}\text { Keynote } \\
\text { Speech II }\end{array}$ & $\begin{array}{l}\text { Prof. Muh. Zamrun } \\
\text { (Rector of Universitas Halu Oleo) }\end{array}$ & Ball Room \\
\hline $11: 30-12: 15$ & $\begin{array}{l}\text { Keynote } \\
\text { Speech III }\end{array}$ & $\begin{array}{l}\text { Prof. Kiril Tenekedjiev (Professor } \\
\text { in Systems Engineering, } \\
\text { University of Tasmania) }\end{array}$ & Ball Room \\
\hline $12: 15-13: 00$ & \multicolumn{3}{|c|}{ Lunch } \\
\hline $13: 00-15: 00$ & Session 1 & $\begin{array}{l}\text { Chairperson: Romi S. Edwin } \\
\text { Tamburaka, Ph.D. }\end{array}$ & Phinisi 3 \\
\hline 13:00-15:00 & Session 2 & $\begin{array}{l}\text { Chairperson: Dr. Eng. Nyoman } \\
\text { Sudiana }\end{array}$ & Azalea \\
\hline
\end{tabular}


ICMID 2018

Kendari, Indonesia 19-20 November 2018

\begin{tabular}{|c|c|c|c|}
\hline 13:00-15:00 & Session 3 & $\begin{array}{l}\text { Chairperson: Hasmina Tari Mokui, } \\
\text { Ph.D. }\end{array}$ & Acacia \\
\hline $15: 00-15: 30$ & \multicolumn{3}{|c|}{ Coffee Break } \\
\hline 15:30-17:00 & Session 4 & Chairperson: Mr. Baso Mursidi & Phinisi 3 \\
\hline $15: 30-17: 00$ & Session 5 & Chairperson: Mr. Masykur Kimsan & Azalea \\
\hline \multirow[t]{2}{*}{$15: 30-17: 00$} & Session 6 & Chairperson: Mr. Al Azis Bahrun & Acacia \\
\hline & \multicolumn{2}{|c|}{$\begin{array}{c}\text { November 20, } 2018 \text { | Tuesday } \\
\text { Grand Clarion Hotel \& Convention Kendari }\end{array}$} & Room \\
\hline 08:00-09:00 & $\begin{array}{l}\text { Invited } \\
\text { Speech I }\end{array}$ & $\begin{array}{l}\text { Dr. Iwan Hermawan } \\
\text { (Earth Observatory of Singapore - } \\
\text { NTU) }\end{array}$ & Ball Room \\
\hline 09:00-10:00 & $\begin{array}{l}\text { Invited } \\
\text { Speech II }\end{array}$ & $\begin{array}{l}\text { Prof. Dr. Ir. Riri Fitri Sari, MM., } \\
\text { M.Sc. (UI Green Metric Program- } \\
\text { Universitas Indonesia) }\end{array}$ & Ball Room \\
\hline $10: 30-11.00$ & \multicolumn{3}{|c|}{ Group Photo \& Coffee Break } \\
\hline 11:00-12:00 & $\begin{array}{l}\text { Invited } \\
\text { Speech III }\end{array}$ & & Ball Room \\
\hline 12:00-13:00 & \multicolumn{3}{|c|}{ Lunch } \\
\hline $13: 00-14: 45$ & Session 7 & $\begin{array}{l}\text { Chairperson: Prof. Dr. Ma'ruf } \\
\text { Kasim }\end{array}$ & Phinisi 3 \\
\hline $13: 00-14: 45$ & Session 8 & $\begin{array}{l}\text { Chairperson: Romi S. Edwin } \\
\text { Tamburaka, Ph.D. }\end{array}$ & Azalea \\
\hline $13: 00-14: 45$ & Session 9 & $\begin{array}{l}\text { Chairperson: Dr. La Ode Muh. } \\
\text { Magribi }\end{array}$ & Acacia \\
\hline $14: 45-15: 30$ & \multicolumn{3}{|c|}{ Coffee Break } \\
\hline $15: 30-17: 00$ & Closing & & Ball Room \\
\hline
\end{tabular}




\section{November 19, 2018, Afternoon}

\section{Monday | Grand Clarion Hotel \& Convention Kendari}

\begin{tabular}{|c|c|}
\hline & $\begin{array}{c}\text { Session } 1 \\
\text { Chairperson: Romi S. Edwin Tamburaka, Ph.D. } \\
\text { Room : Phinisi } 3\end{array}$ \\
\hline $13: 00-13: 15$ & $\begin{array}{l}\text { [ICMID18-10SC] Characteristics of Quaternary Deep Sea } \\
\text { Sediments Based on Grain size Analysis and LOI (Lost of ignition) } \\
\text { on the Sumba Strait of East Nusa Tenggara } \\
\text { Zulhikmah, Yuniarti Yuskar, Purna Sulastya Putra, Septriono Hari } \\
\text { Nugroho and Tiggi Choanji } \\
\text { Universitas Islam Riau, Indonesian Institute of Sciences (LIPI), } \\
\text { Bandung, Indonesia, Indonesian Institute of Sciences (LIPI), } \\
\text { Ambon, Indonesia }\end{array}$ \\
\hline $13: 15-13: 30$ & $\begin{array}{l}\text { [ICMID18-18SC] Characterization of Liquid Volatile Matter Rice } \\
\text { Husk Using Gas Chromatography } \\
\text { Wa Ode Sitti Ilmawati, Muhammad Jahiding Mashuni, and Kinanti } \\
\text { Universitas Halu Oleo, Indonesia }\end{array}$ \\
\hline $13: 30-13: 45$ & $\begin{array}{l}\text { [ICMID18-22SC] Hybrid Method Analysis of Variance (ANOVA) } \\
\text { and Loss Function to Analyzed Optimizing Composition of Fiber } \\
\text { Baggase and Fly Ash } \\
\text { L Laome, M Hasbi } \\
\text { Universitas Halu Oleo, Indonesia }\end{array}$ \\
\hline $13: 45-14: 00$ & $\begin{array}{l}\text { [ICMID18-34SC] Composition Chemistry And Distribution to } \\
\text { Quality of Essential Oil From Rhizome E.elatior and E.calophyris } \\
\text { By Gas Chromatography-Mass Spectrometry (GC-MS) } \\
\text { Muhammad Aswan, Nur Arafah, I. Sahidin, Imran, Suryani, L.O. } \\
\text { Ngkoimani } \\
\text { Universitas Halu Oleo, Indonesia }\end{array}$ \\
\hline $14: 15-14: 30$ & $\begin{array}{l}\text { [ICMID18-7SC] Stability Analysis and Reinforcement on Wall of } \\
\text { Seropan Cave in Karst Zone } \\
\text { Sulha, Ahmad Rifa'I, Minson Simatupang and Umran Sarita } \\
\text { Universitas Halu Oleo, Indonesia }\end{array}$ \\
\hline
\end{tabular}


ICMID 2018

Kendari, Indonesia 19-20 November 2018

14:30-14:45 [ICMID18-45AE] Effect of Current Density on Hardness of Low Carbon Steel Electroplated by Copper, Nickel and Copper-Nickel

Sudarsono Sudarsono, Irma Nurjannah and Aminur Aminur Universitas Halu Oleo, Indonesia

14.45-15.00 [ICMID18-55SC] Groundwater change detection by gravity measurement on northern coast of Java: a case study in Semarang City of Central Java of Indonesia

Laode M Sabri, Bambang Sudarsono, Yadi Aryadi, Dwi Novianto Universitas Diponegoro, Badan Informasi Geospasial, Indonesia

$15: 00-15: 30$ Coffee Break

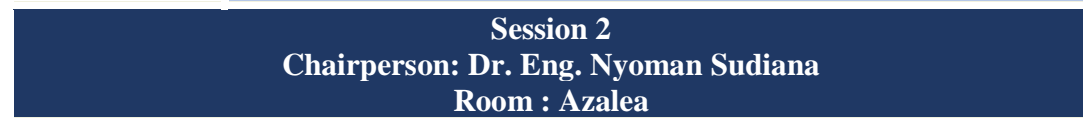

13:00-13:15 [ICMID18-42AE] Design of A Prototype System for Monitoring Air Quality for Smart City Implementation

Yuni Aryani Koedoes, Samue Jie, Muh. Nadzirin Anshari Nur, Bunyamin

Universitas Halu Oleo, Indonesia

13:15-13:30 [ICMID18-39AE] The Concept of Aquarium Building Design and the Ornamental Fish Market in Muara Baru: Through the Process of Healing Urban Acupuncture

Eric Subroto, Fermanto Lianto

Universitas Tarumanegara, Indonesia

13:30-13:45] [ICMID18-19PO] Floating Market in Muara Angke

Mieke Choandi, Lourensya Jessica Berlian

Universitas Tarumanegara, Indonesia

13:45-14:00 [ICMID18-36AE] Floating Houses Technology as Alternative Living on The Water

Tri Endangsih, Ikaputra

Universitas Budi Luhur, Universitas Gadjah Mada, Indonesia

14:15-14:30 [ICMID18-13PO] Community Based Tourism for the Concept of Village Tourism Facility Development in Papan Island 
ICMID 2018

Kendari, Indonesia 19-20 November 2018

Irdinal Arief and Harifuddin Thahir

Universitas Tadulako, Indonesia

14:30-14:45 [ICMID18-28PO] Carrying Capacity of Nambo Beach Tourism

Destination in Kendari City as Implementation of National and

Regional Tourism Policies

Sitti Hairani Idrus, La Ode Muh. Golok Jaya

Universitas Halu Oleo, Indonesia

14:45-15:00 [ICMID18-24PO] Analysis of Torobulu - Tampo Port Service Performance to Improve Mobility of People and Good Movements

Adris A. Putra, Edward Ngii, Rudi Balaka, Mappa Nashrun, Harsawan

Universitas Halu Oleo, Indonesia

15:00-15:30 Coffee Break

Session 3
Chairperson: Hasmina Tari Mokui, Ph.D.
Room : Acacia

13:00-13:15 [ICMID18-38AE] The Analysis of Work Acceleration in Tower Construction of Sultra Bank Using Fast Track Method and Cost Trade Time Off

Nur Hizrah, Susanti Djalante, Fitriah Mashud

Universitas Halu Oleo, Indonesia

13:15-13:30 [ICMID18-23SC] Analysis of Longshore Sediment Transport at The Estuaries of Jeneberang River and Tallo River Caused by Waves on Coast of Makassar

Riswal Karamma, M.Saleh Pallu, M.Arsyad Thaha, Mukhsan Putra Hatta, A.Subhan Mustari, Ahmad Syarif Sukri

Universitas Hasanuddin, Universitas Halu Oleo, Indonesia

13:30-13:45 [ICMID18-12SC] Technical Feasibility Analysis of Waode BuriLelamo Port as Local Feeder Port in North Buton Regency Southeast Sulawesi Province

S Ramadan, I Kadir, M Z Umar and L M N Arsyad Universitas Halu Oleo, Indonesia 
ICMID 2018

Kendari, Indonesia 19-20 November 2018

13:45-14:00 [ICMID18-51SC] The magnetic susceptibility of undeformed dacite intrusions within the Kolaka fault zone, SE Sulawesi, Indonesia

L.O. Ngkoimani, Jahidin, Suryawan Asfar, La Hamimu, Harisma Buburanda

Universitas Halu Oleo, Indonesia

14:15-14:30 [ICMID18-53SC] Proximate analysis of coal quality from Uluiwoi District, Kolaka Timur Regency, Southeast Sulawesi Province

Jahidin, Harisma Buburanda, Suryawan Asfar, La Hamimu, La Ode Ngkoimani

Universitas Halu Oleo, Indonesia

14:30-14:45 [ICMID18-43SC] Shear Strength Analysis of Reduced Beam Section (RBS) on Castellated Beam

Nini Hasriyani Aswad, Tachrir, Herman Parung, A. Arwin Amiruddin

Universitas Halu Oleo, Universitas Hasanuddin, Indonesia

14:45-15:00 [ICMID18-49SC] The Correlation of Magnetic Susceptibility and $\mathrm{Fe}, \mathrm{Zn}, \mathrm{Mn}$, Content of Topsoil Samples and its Relationship with Land Use Activities in Kendari City

La Ode Ngkoimani, Hasriani, Ramlia, La Ode Safiuddin, Jahidin Universitas Halu Oleo, Indonesia

15:00-15:30 Coffee Break

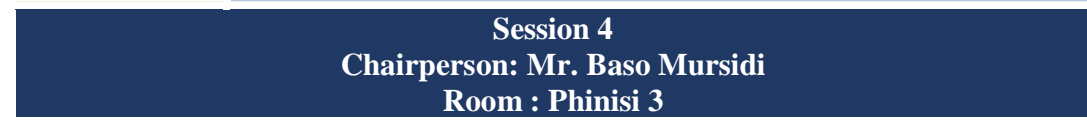

15:30-15:45 [ICMID18-29AE] Carbon Stocks Mapping of Mangrove Forest in North Buton Indonesia using Combination of Optical and Radar Imagery in the Perspective of Climate Change Mitigation

Laode M Golok Jaya, Muhammad Yamin, Ika Purwanti Ningrum, Jamhir Safani, Sitti Hairani Idrus and Ketut Wikantika Universitas Halu Oleo, Institut Teknologi Bandung, Indonesia

15:45-16:00 [ICMID18-32SC] Fuzzy Logic Methods to Identify Potential Area Mapping for Mangrove Forests in Kendari Using Landsat Image 
ICMID 2018

Kendari, Indonesia 19-20 November 2018

$16: 00-16: 15$

$16: 15-16: 30$

$16: 30-16: 45$

$16: 45-17: 00$
Ika Purwanti Ningrum, Sutardi, Rizal Adi Saputra, Adha Mashur Sajiah, Syamsul Razak Haraty, Hasran Jaya Universitas Halu Oleo, Indonesia

[ICMID18-17AE] Geothermal Energy Mapping Based on Land Surface Temperature Analysis of Remote Sensing Satellite Imagery (Case Study: North Konawe District)

Laode M Golok Jaya and Andri Suprayogi

Universitas Halu Oleo, Universitas Diponegoro, Indonesia

[ICMID18-31SC] Fuzzy Analysis to Determine Potential Catching Areas of Skipjack Tuna on Southeast Sulawesi Waters

Ika Purwanti Ningrum, Rizal Adi Saputra, Jayanti Yusmah Sari, Mutmainnah Muchtar, Ismail Yushar

Universitas Halu Oleo, Indonesia

[ICMID18-5AE] Analysis morphometric and morphotectonic DAM Pelosika Using Remote Sensing and GIS Method

Suryawan Asfar, Andi Makkawaru and Sayahdin Alfat

[ICMID18-30AE] Geometric Accuracy Assesment Of Orthorectification Method Based On Sensor Model Refinement In Open Source System Environment (Case Study: Sangir Subdistrict, South Solok District, West Sumatra Province)

Andri Suprayogi and La Ode Muh. Golok Jaya

Universitas Diponegoro, Universitas Halu Oleo, Indonesia

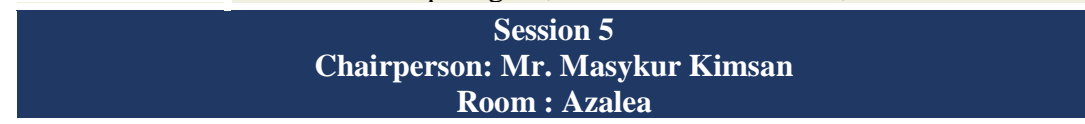

15:30-15:45 [ICMID18-21PO] Coastal Landuse Change and The Need of Greenspace Based Development Coastal Area Settlement of South Sulawesi

Husni Kotta, M, Arman Faslih, La Ode Amrul Hasan Universitas Halu Oleo, Indonesia

15:45-16:00 [ICMID18-9SC] Modern Analogue of Shaly Sand Reservoir on Coastal Plain in Selat Baru, Bengkalis Island, Indonesia 
ICMID 2018

16:00-16:15 [ICMID18-11PO] Modernization of Seaweed Cultivation Method As one of the strategies to increase farmers' income

Ma'ruf Kasim, Budiyanto, Sarini Djusuf Abadi

Universitas Halu Oleo, Indonesia

16:15-16:30 [ICMID18-4PO] Sustainability of Sulaa Coastal Areas as Heritage Assets of Baubau in Southeast Sulawesi

Ishak Kadir, Sachrul Ramadhan and Muhammad Zakaria Umar Universitas Halu Oleo, Indonesia

16:30-16:45 [ICMID18-8AE] Effect of Water Content in Changing the Bearing Capacity of Subgrade (Case Study: Kendari-Moramo Road, Konawe Selatan)

Fitriah Mas’ud, Sulha, Romy S.Edwin, Umran Sarita, Ridwansyah Nuhun, Mega Dwi Anita

Universitas Halu Oleo, Indonesia

16:45-17:00 [ICMID18-56SC] Microwave Processing Silica Ceramic Derived from Rice Husk Ash

I N Sudiana, L Aba, L Lestari, H Aripin, M Z Firihu, S Mitsudo Universitas Halu Oleo, Universitas Siliwangi, Indonesia, University of Fukui, Japan

Session 6
Chairperson: Mr. Al Azis Bahrun
Room : Acacia

15:30-15:45 [ICMID18-26AE] Potential use of pomalaa nickel slag as a substitute for sand in brick making

Edward Ngii, BasoMursidi, Yogi Yudhistira Umar

Civil Engineering Dept., Faculty of Engineering, Universitas Halu Oleo, Indonesia

15:45-16:00 [ICMID18-50AE] Evaluation of bottom ash composition on modified hollow brick design with sago husk as filler 
ICMID 2018

Kendari, Indonesia 19-20 November 2018

Kurniati Ornam, Masykur Kimsan, La Ode Ngkoimani, Siti Belinda Amri, Santi, and Syafrianto Amsyar Universitas Halu Oleo, Indonesia

16:00-16:15 [ICMID18-6SC] Numerical Investigation of Structural Analysis on Sandwich Material Using Finite Element Method

Sayahdin Alfat

Universitas Halu Oleo, Indonesia

16:15-16:30 [ICMID18-52AE] Performance level evaluation of modified hollow bricks utilization for housing

Masykur Kimsan, Kurniati Ornam, Siti Belinda Amri, Santi, and Syafrianto Amsyar

Universitas Halu Oleo, Indonesia

16:30-16:45] [ICMID18-40SC] Effect of Compaction Pressure on Quality of Activated Charcoal Briquette Made from Sago Stem Midrib Material

L Lestari, V I Variani, M Z Firihu, S Raharjo, I Saleh, and Nining Aprilla

Universitas Halu Oleo, Indonesia

16:45-17:00 [ICMID18-3AE] Concrete Brick Material from Nambo Sea Sand and Nambo Excavated Sand

Arman Faslih and Muhammad Zakaria Umar

Universitas Halu Oleo, Indonesia 


\section{November 20, 2018, Afternoon}

Tuesday | Grand Clarion Hotel \& Convention Kendari

\begin{tabular}{|c|c|}
\hline & $\begin{array}{c}\text { Session } 7 \\
\text { Chairperson: Prof. Dr. Ma'ruf Kasim } \\
\text { Room : Phinisi } 3\end{array}$ \\
\hline $13: 00-13: 15$ & $\begin{array}{l}\text { [ICMID18-1SC] Transportasion Demand Analysis Using Taxy } \\
\text { Mode in Kendari City } \\
\text { La Ode Muhammad Magribi, Susanti Djalante,Muhammad Anas } \\
\text { Pradiharja } \\
\text { Universitas Halu Oleo, Indonesia }\end{array}$ \\
\hline $13: 15-13: 30$ & $\begin{array}{l}\text { [ICMID18-15PO] Performance and Perception of User Trans Lulo } \\
\text { Service Users in Kendari City } \\
\text { Laode Mahdiyanto Samara, Laode Muhammad Magribi, Susanti } \\
\text { Djalante } \\
\text { Universitas Halu Oleo, Indonesia }\end{array}$ \\
\hline $13: 30-13: 45$ & $\begin{array}{l}\text { [ICMID18-25PO] Cost Analysis of Traffic Accidents and Loss of } \\
\text { Value of Productivity Victims of Traffic Accident Personal } \\
\text { Vehicle with Four Wheels of Time Value in Kendari City } \\
\text { Nasrul, Rudi Balaka, Multi Bahari La Ode, Muammar Makmur } \\
\text { Universitas Halu Oleo, Indonesia }\end{array}$ \\
\hline $13: 45-14: 00$ & $\begin{array}{l}\text { [ICMID18-16PO] Assesing The Peformance of Passenger } \\
\text { Transport Service Terminal in Kendari City } \\
\text { Rudi Balaka, Ridwansyah Nuhun, Thahir Azikin and Laode M. } \\
\text { Agum Gumelar } \\
\text { Universitas Halu Oleo, Indonesia }\end{array}$ \\
\hline $14: 15-14: 30$ & $\begin{array}{l}\text { [ICMID18-27AE] Service Desk Application (SDA) as Media to } \\
\text { Improve Maintenance Services and improvements of Campus } \\
\text { Assets in Polytechnic State of Cilacap }\end{array}$ \\
\hline $14: 30-14: 45$ & $\begin{array}{l}\text { Riyadi Purwanto, Muh Yamin, Laode M Golok Jaya, Yuspian } \\
\text { Gunawan } \\
\text { Politeknik Negeri Cilacap, Universitas Halu Oleo, Indonesia } \\
\text { [ICMID18-57SC] The Effect of Magnesium Oxide (MgO) on } \\
\text { Properties of High Purity Alpha Alumina }\end{array}$ \\
\hline
\end{tabular}


ICMID 2018

Kendari, Indonesia 19-20 November 2018

I N Sudiana, L Aba, H Aripin, K G Suastika, S Mitsudo, M Z Firihu Universitas Halu Oleo, Universitas Siliwangi, Universitas Palangkaraya, Indonesia

University of Fukui, Japan

\section{Session 8}

Chairperson: Romi S. Edwin Tamburaka, Ph.D.

Room : Azalea

13:00-13:15 [ICMID18-37PO] Feasibility Study for Renewable Energy Based

Micro Grid System for Kabaena Island South East Sulawesi

Mansur, Salama Manjang, Ardiaty Arief, Yusri Syam Akil

Universitas Hasanuddin, Universitas Halu Oleo, Indonesia

13:15-13:30 [ICMID18-20SC] The Potential of Plastic Waste for Utilizing into Pyrolysis Oil Have to Support Independent Energy Communities in Kendari City

Yuspian Gunawan, La Karimuna, La Ode Magribi, Lukas Kano Mangalla, Ridway Balaka, Mustarum Musaruddin, Salimin, Jenny Delly, Bunyamin, Abdul Johar, Ahmad Syarif Syukri, Aminur, Prinop Aksar

Universitas Halu Oleo, Indonesia

13:30-13:45 [ICMID18-46PO] Analysis of Islamic Center Planning with Ecological Architecture Approach in Kendari City

Adelila Haksika Fisika Ratna and Irma Nurjannah Universitas Halu Oleo, Indonesia

13:45-14:00 [ICMID18-48PO] Space Utilization Optimization New Campus of Halu Oleo University

La Ode Amrul Hasan, Yudhi Dwi Hartono, Arif Saleh, Alim Bahri Universitas Halu Oleo, Universitas Muhammadiyah Kendari, Indonesia

14:15-14:30 [ICMID18-44SC] Dynamic Behavior of Human Tympanic Membrane Perforation Using Finite Element Method

Hidayat Hidayat, Sudarsono Sudarsono and Rozaini Othman Politeknik Negeri Samarinda, Universitas Halu Oleo, Indonesia, Unversiti Teknologi MARA Pulau Pinang, Malaysia 
ICMID 2018

Kendari, Indonesia 19-20 November 2018

$14.30-14.45$

[ICMID18-54PO] The Study of Dynamic of Coastal Resource

Community-Based Management in Wabula Village of Buton

Regency

La Nalefo, Bahtiar, Salahuddin, and Ine Fausayana

Universitas Halu Oleo, Indonesia

\begin{tabular}{|c|c|}
\hline & $\begin{array}{c}\text { Session } 9 \\
\text { Chairperson: Dr. La Ode Muh. Magribi } \\
\text { Room : Acacia }\end{array}$ \\
\hline \multirow[t]{2}{*}{$13: 00-13: 15$} & $\begin{array}{l}\text { [ICMID18-35AE] Design and Development of Automatic Main } \\
\text { Failure (AMF) Control System Using Plc Based SMS }\end{array}$ \\
\hline & $\begin{array}{l}\text { Sry Defi, Luther Pagiling, Muh. Nadzirin Anshari Nur } \\
\text { Universitas Halu Oleo, Indonesia }\end{array}$ \\
\hline \multirow[t]{2}{*}{$13: 15-13: 30$} & $\begin{array}{l}\text { [ICMID18-41AE] Turbine Design of Electricity Power Plant } \\
\text { Using Oscillating Water Column Technology Using Ansys } 16.0 \\
\text { Program }\end{array}$ \\
\hline & $\begin{array}{l}\text { Jenny Delly, Al Ichlas Imran, Baso Mursidi } \\
\text { Universitas Halu Oleo, Indonesia }\end{array}$ \\
\hline \multirow[t]{2}{*}{$13: 30-13: 45$} & $\begin{array}{l}\text { [ICMID18-33SC] Experimental Study on The Performance } \\
\text { Characteristics of a Microwave - Solar Heating Dryer }\end{array}$ \\
\hline & $\begin{array}{l}\text { Mangalla L.K., Lolok A., Delly J., Kadir, Samhuddin, Kadir A., } \\
\text { Sudia B., Pagiling L. } \\
\text { Universitas Halu Oleo, Indonesia }\end{array}$ \\
\hline \multirow[t]{2}{*}{$13: 45-14: 00$} & $\begin{array}{l}\text { [ICMID18-14AE] Smart kWh Meter for Improved Power System } \\
\text { Security and Resiliency }\end{array}$ \\
\hline & $\begin{array}{l}\text { Barakah Aswad, Fitra Rachmad Tullah, Dudi Rahman, Hasmina } \\
\text { Tari Mokui } \\
\text { Universitas Halu Oleo, Indonesia }\end{array}$ \\
\hline $14: 15-14: 30$ & $\begin{array}{l}\text { [ICMID18-47AE] Application of Under-Frequency Relay (UFR) } \\
\text { in Load Shedding Scheme for System Stability (A Case Study on } \\
\text { PT. PLN Kendari) }\end{array}$ \\
\hline & $\begin{array}{l}\text { Tachrir, Samuel Jie, Bunyamin, Abdul Djohar } \\
\text { Universitas Halu Oleo, Indonesia }\end{array}$ \\
\hline
\end{tabular}


ICMID 2018

Kendari, Indonesia 19-20 November 2018

14:30-14:45 [ICMID18-2SC] A Dimensionless Perspective of Thermal Effect Variations on Crack Growth Using Adaptive Finite Element Method in Homogeneous Material

Sayahdin Alfat, Masato Kimura and Suryawan Asfar Universitas Halu Oleo, Indonesia 


\section{Table of Contents}

Foreword from ICMID Chairperson ___

Foreword from Dean of Faculty of Engineering UHO __ iii

Technical Program__ v

\section{Session 1}

[ICMID18-10SC] Characteristics of Quaternary Deep Sea Sediment in the Sumba Strait based on Grain Size and Lol (Lost on ignition Analysis) _ 1 [ICMID18-18SC] Characterization of Liquid Volatile Matter Rice Husk Using Gas Chromatography 2

[ICMID18-22SC] Hybrid Method Analysis of Variance (ANOVA) and Loss Function to Analyzed Optimizing Composition of Fiber Baggase and Fly Ash

[ICMID18-34SC] Composition Chemistry and Distribution to Quality of Essential Oil From Rhizome E.elatior and E.calophyris By Gas Chromatography-Mass Spectrometry (GC-MS) 4

[ICMID18-7SC] Stability Analysis and Reinforcement On Wall Of Seropan Cave In Karst Zone 5

[ICMID18-45AE] Effect of Current Density on Hardness of Low Carbon Steel Electroplated by Copper, Nickel and Copper-Nickel 6

[ICMID18-55SC] Groundwater change detection by gravity measurement on northern coast of Java: a case study in Semarang City of Central Java of Indonesia

\section{Session 2}

[ICMID18-42AE] Design of Prototype System for Monitoring Air Quality for Smart City Implementation 
[ICMID18-39AE] The Concept of Aquarium Building Design and the Ornamental Fish Market in Muara Baru: Through the Process of Healing Urban Acupuncture

[ICMID18-19PO] Floating Market in Muara Angke 10

[ICMID18-36AE] Floating Houses Technology as Alternative Living on The Water

[ICMID18-13PO] Community Based Tourism for the Concept of Village Tourism Facility Development in Papan Island 12

[ICMID18-28PO] Carrying Capacity of Nambo Beach Tourism Destination in Kendari City as Implementation of National and Regional Tourism Policies 13

[ICMID18-24PO] Analysis of Torobulu - Tampo Port Service Performance to Improve Mobility Of People And Good Movements 14

\section{Session 3}

[ICMID18-38AE] The Analysis of Work Acceleration In Tower Construction of Sultra Bank Using Fast Track Method And Cost Trade Time Off

[ICMID18-23SC] Analysis of Longshore Sediment Transport at Theestuaries of Jeneberang River and Tallo River Caused by Waves on Coast of Makassar 16

[ICMID18-12SC] Technical Feasibility Analysis of Waode Buri-Lelamo Port as Local Feeder Port in North Buton Regency Southeast Sulawesi Province

[ICMID18-51SC] The Magnetic Susceptibility of Undeformed Dacite Intrusions Within the Kolaka Fault Zone, SE Sulawesi, Indonesia 18 [ICMID18-53SC] Proximate analysis of coal quality from Uluiwoi District, Kolaka Timur Regency, Southeast Sulawesi Province 19 
[ICMID18-43SC] Shear Strength Analysis of Reduced Beam Section (RBS) on Castellated Beam 20 [ICMID18-49SC] The Correlation of Magnetic Susceptibility and Fe, Zn, $\mathrm{Mn}$, Content of Topsoil Samples and its Relationship with Land Use Activities in Kendari City

\section{Session 4}

[ICMID18-29AE] Carbon Stocks Mapping of Mangrove Forest in North Buton Indonesia using Combination of Optical and Radar Imagery in the Perspective of Climate Change Mitigation 22

[ICMID18-32SC] Fuzzy Logic Methods to Identify Potential Area Mapping for Mangrove Forests in Kendari using Landsat Image 24

[ICMID18-17AE] Geothermal Energy Mapping Based on Land Surface Temperature Analysis of Remote Sensing Satellite Imagery (Case Study: North Konawe District) 25

[ICMID18-31SC] Fuzzy Analysis to Determine Potential Catching Areas of Skipjack Tuna on Southeast Sulawesi Waters 27 [ICMID18-5AE] Analysis Morphometric and Morphotectonic DAM Pelosika Using Remote Sensing and GIS Method 28

[ICMID18-30AE] Geometric Accuracy Assessment of Ortho-rectification Method Based on Sensor Model Refinement In Open Source System Environment (Case Study: Sangir Subdistrict, South Solok District, West Sumatra Province) 29

\section{Session 5}

[ICMID18-21PO] Coastal Landuse Change and The Need of Greenspace Based Development Coastal Area Settlement of South Sulawesi 31 
[ICMID18-9SC] Modern Analogue of Shaly Sand Reservoir on Coastal Plain in SelatBaru, Bengkalis Island, Indonesia 32 [ICMID18-11PO] Modernization of Seaweed Cultivation Method As one of the strategies to increase farmers' income 33 [ICMID18-4PO] Sustainability of Sulaa Coastal Areas as Heritage Assets in Baubau City Southeast Sulawesi Province 34 [ICMID18-8AE] Effect of Water Content in Changing The Bearing Capacity of Subgrade (Case Study: Kendari-Moramo Road, Konawe Selatan) [ICMID18-56SC] Microwave Processing Silica Ceramic Derived from Rice Husk Ash 36

\section{Session 6}

[ICMID18-26AE] Potential Use of Pomalaa Nickel Slag as A Substitute for Sand in Brick Making

[ICMID18-50AE] Evaluation of Bottom Ash Composition on Modified Hollow Brick Design with Sago Husk as Filler 38

[ICMID18-6SC] Numerical Investigation of Structural Analysis on Sandwich Material Using Finite Element Method 39 [ICMID18-52AE] Performance Level Evaluation of Modified Hollow Bricks Utilization for Housing 40 [ICMID18-40SC] Effect of Compaction Pressure on Quality of Activated Charcoal Briquette Made from Sago Stem Midrib Material 41 [ICMID18-3AE] Concrete Brick Material from Nambo Sea Sand and Nambo Excavated Sand 42 


\section{Session 7}

[ICMID18-1SC] Transportasion Demand Analysis Using Taxy Mode in Kendari City 43

[ICMID18-15PO] Performance and Perception of User Trans Lulo Service Users in Kendari City 45 [ICMID18-25PO] Cost Analysis of Traffic Accidents and Loss of Value of Productivity Victims of Traffic Accident Personal Vehicle with Four Wheels of Time Value in Kendari City 46

[ICMID18-16PO] Assesing The Peformance Of Passenger Transport Service Terminal in Kendari City 47

[ICMID18-27AE] Service Desk Application (SDA) as Media to Improve Maintenance Services and improvements of Campus Assets in Polytechnic State of Cilacap 48

[ICMID18-57SC] The Effect of Magnesium Oxide (MgO) on Properties of High Purity Alpha Alumina 50

\section{Session 8}

[ICMID18-37PO] Feasibility Study for Renewable Energy Based Micro Grid System for Kabaena Island South East Sulawesi

[ICMID18-20SC] The Potential of Plastic Waste for Utilizing into Pyrolysis Oil Have to Support Independent Energy Communities in Kendari City 52

[ICMID18-46PO] Analysis of Islamic Center Planning with Ecological Architecture Approach in Kendari City 53

[ICMID18-48PO] Space Utilization Optimazation New Campus of Halu Oleo University 54

[ICMID18-44SC] Dynamic Behavior of Human Tympanic Membrane Perforation Using Finite Element Method 55 
[ICMID18-54PO] The Study of Dynamic of Coastal Resource CommunityBased Management in Wabula Village of Buton Regency 56

\section{Session 9}

[ICMID18-35AE] Design and Development of Automatic Main Failure (AMF) Control System Using PLC Based SMS

[ICMID18-41AE] Turbine Design of Electricity Power Plant Using Oscillating Water Column Technology Using Ansys 16.0 Program 58 [ICMID18-33SC] Experimental Study on the Performance Characteristics of A Microwave - Solar Heating Dryer 59 [ICMID18-14AE] Smart kWh Meter for Improved Power System Security and Resiliency 60 [ICMID18-47AE] Application of Under-Frequency Relay (UFR) in Load Shedding Scheme for System Stability (A Case Study on PT. PLN Kendari)

[ICMID18-2SC] A Dimensionless Perspective of Thermal Effect Variations on Crack Growth Using Adaptive Finite Element Method in Homogeneous Material 62 